\title{
A Novel Sensor for Attitude Determination Using Global Positioning System Signals
}

\author{
John L. Crassidis ${ }^{1}$ \\ David A. Quinn ${ }^{2}$ \\ F. Landis Markley ${ }^{2}$ \\ Jon D. McCullough ${ }^{2}$
}

\begin{abstract}
An entirely new sensor approach for attitude determination using Global Positioning System (GPS; signals is developed. The concept involves the use of multiple GPS antenna elements arrayed on a single sensor head to provide maximum GPS space vehicle availability. A number of sensor element configurations are discussed. In addition to the navigation function, the array is used to find which GPS space vehicles are within the field-of-view of each antenna element. Attitude determination is performed by considering the sightline vectors of the found GPS space vehicles together with the fixed boresight vectors of the individual antenna elements. This approach has clear advantages over the standard differential carrier-phase approach. First, errors induced by multipath effects can be significantly reduced or eliminated altogether. Also, integer ambiguity resolution is not required, nor do line biases need to be determined through costly and cumbersome self-surveys. Furthermore, the new sensor does not require individual antennas to be physically separated to form interferometric baselines to determine attitude. Finally, development potential of the new sensor is limited only by antenna and receiver technology development unlike the physical limitations of the current interferometric attitude determination scheme. Simulation results indicate that accuracies of about 1 degree $(3 \sigma)$ are possible.
\end{abstract}

\footnotetext{
'Assistant Professor, Catholic University of America, Dept. of Mech. Eng., Washington. D.C. 20064.

'Engineer, Goddard Space Flight Center, Guidance, Navigation. and Control Center, Greenbelt, MD 20771.
} 


\section{Introduction}

The Global Positioning System (GPS) constellation was originally developed to permit a wide variety of user vehicles an accurate means of determining position for autonomous navigation. The constellation includes 24 space vehicles (SVs) in semi-synchronous (12 hour) orbits, providing a minimum of six SVs in view for ground-based navigation. The underlying principle involves geometric triangulation with the GPS SVs as known reference points to determine the user's position to a high degree of accuracy. The GPS was originally intended for ground-based and aviation applications, gaining much attention in the commercial community (e.g., automobile navigation, aircraft landing, etc.). However, in recent years there has been a growing interest in space-based applications. Since the GPS SVs are in approximately $20,000 \mathrm{~km}$ circular orbits, the position of any potential user below the constellation may be easily determined. A minimum of four SVs are required so that in addition to the three-dimensional position of the user, the time of the solution can be determined and in turn employed to correct the user's clock. Since its original inception, there have been many innovative improvements to the accuracy of the GPS determined position. These include using local area as well as wide area differential GPS, carrier-phase differential GPS, and so-called "pseudolites" (ground-based GPS transmitters) [1]. In particular, carrier-phase differential GPS measures the phase of the GPS carrier relative to the phase at a reference site, which dramatically improves the position accuracy [2]. Also, for spacecraft applications dynamically aided GPS using orbit models with GPS measurements in an extended Kalman filter can improve position accuracy.

Early applications of this concept to user spacecraft in Low Earth Orbits (LEOs) have demonstrated extremely useful results [3]. Recently, there have been investigations of position determination by user spacecraft from above the GPS constellation [4]. Since current GPS SVs transmit their signals towards the Earth, this concept poses a much more difficult problem because the user spacecraft must rely on "spillage" signals received from GPS SVs on the far side of the Earth.

Another aspect of space-based applications using GPS that has gained much recent attention is attitude determination. One of the first space-based applications was flown on the RADCAL (RADar CALibration) spacecraft [5], which demonstrated a GPS attitude determination capability using postprocessed measurements. To obtain maximum GPS visibility, and to reduce signal interference due to multipath reflection, GPS patch antennas were placed on the top surface of the spacecraft bus. Although the antenna baselines were relatively short for attitude determination ( 0.67 meter separation), attitude 
accuracy on the order of 2 degrees per axis $(3 \sigma)$ was achieved. Another experiment, Crista-SPAS [6] provided the first on-orbit demonstration of real-time attitude determination. The spacecraft contained an accurate gyro reference, but the coordinate frame alignment was not measured relative to the GPS attitude reference frame, which means that discrepancies between the two reference frames might account for slightly different measurements from the two systems. Over the course of the experiment, the two sets of attitude solutions agreed to within 2 degrees, which was thought to be within the alignment tolerance of the two reference frames. The first extended real-time GPS based attitude determination mission was flown on the REX-II spacecraft [7], which tested actual attitude control using GPS attitude measurements.

The differential carrier-phase measurement error has a standard deviation of about 10 degrees, a small fraction of the standard wavelength [8]. However, many error sources can significantly contribute to attitude inaccuracy. These include: reflections of the GPS carrier from the environment surrounding the antennas (multipath), electrical dissipation inherent when passing carrier-phase signals over the lengths of the RF cables between antennas and receiver (line bias errors), antenna motions due to external disturbances (e.g., thermal distortion effects), constellation availability, tropospheric refraction, and cross-talk errors. The most significant error source and the most difficult to overcome is multipath [9]. In fact, multipath effects can be so pronounced as to be a major driver for the location of the GPS antennas on a vehicle. Despite limited successes with recent attempts at modeling multipath [10], this error remains a limiting factor in the performance of carrier-phase based GPS attitude determination. This is due to the complex physical nature of the reflecting surfaces, which depends mostly on antenna locations. Line biases can also adversely affect carrier-phase based attitude. These biases are typically determined by performing extensive calibrations (self survey) of the flight system on the ground prior to launch. However, since the space environment can significantly alter the physical properties of the cable through large temperature gradients, a permanent solution to this problem remains elusive. Yet another error source for the carrier-phase based method involves shifting baselines. In general, the attainable attitude accuracy improves with longer baselines. If, however, satisfactorily separating the GPS antennas requires mounting them on flexible structures (such as solar arrays, or deployable booms), then the attitude performance of the carrier-phase based method can be seriously compromised to the point where the advantages of the longer baseline is compromised. It is important to recognize that the 
aforementioned errors are primarily a result of the physical problems associated with using carrier-phase based measurements for attitude determination.

Before the actual GPS attitude determination can be performed, the correct number of integer wavelengths between each pair of antennas must be found. The resolution of these integer ambiguities has been extensively investigated [11]. Such integer resolution techniques fall into two general categories: instantaneous and motion-based techniques. Instantaneous techniques provide immediate integer resolution without vehicle motion; however, the uniqueness of the solution may be severely degraded with sensor noise. Motion-based techniques use a batch of data to determine the integers; however, they rely on sufficient vehicle motion to obtain system observability. In either case, it is essential that these integers are accurately resolved before attitude determination can occur.

In this paper, a new sensor approach for GPS attitude determination is shown. This essentially involves using an array of GPS antenna positioned to provide maximum sky coverage. This array is used only to find which GPS spacecraft are within the field-of-view (FOV) of each antenna. Attitude determination is performed by considering the sightline vectors of the found GPS spacecraft together with the boresight vector of the particular antenna, unlike interferometric methods (see Refs. [12]-[14]). The boresight is used since the exact location of the GPS spacecraft in the body-frame of the antenna FOV is not known. The approach essentially is similar to a star tracker, with the GPS sightline vectors as the inertial reference vectors and the antenna boresight vectors as the body vectors. Multiple antennas are used to increase attitude accuracy. The advantages of the new sensor approach include: 1) differential carrier-phase measurements are not required, 2) attitude errors from multipath can be reduced or even eliminated, 3 ) integer ambiguities do not need to be resolved, and 4) line biases do not need to be determined. Therefore, the new sensor approach is easy to implement and use for any application. It will be shown that the accuracy of the new sensor is better as the FOV decreases. Multiple sensor configurations are tested to investigate this concept. Even though the accuracy in simulations is currently not better than the standard carrier-phase approach, the new sensor is only limited by technology. As technology advances, more GPS antenna can be used to further increase attitude accuracy.

The organization of this paper proceeds as follows. First, the new sensor concept is shown. A number of antenna configurations are shown. Next, a review of Wahba's problem is shown for attitude 
determination, as well as a method to determine the associated weights in the loss function. Simulation results are then presented, with a discussion of the procedures for actual hardware implementation.

\section{New GPS Sensor Concept}

In this section the concept of the Compound Eye GPS Attitude and Navigation Sensor (CEGANS) is introduced. A number of sensor configurations are shown for the new sensor. Next, an attitude determination algorithm is developed, which is accomplished by expanding upon current efficient methods. Finally, simulation results are presented.

The Compound Eye GPS Attitude Sensor

The basic concept underlying the CEGANS is a relatively simple one that uses GPS antennas in way similar to methods employed by star trackers for many years. When considering GPS for navigation uses only, it is advantageous for a single antenna to cover as much of the visible sky as possible, allowing signals from as many GPS SVs to be processed as are available to the user. In this way, the best possible navigation solution can be ascertained with the minimum amount of spacecraft hardware. The natural result of this approach has been the development of patch antennas capable of tracking GPS SVs over a hemispherical FOV.

An ideal solution is to provide an attitude capability without losing the navigation function, while simultaneously avoiding the constraints and requirements imposed by the interferometric method discussed above. This is an approachable goal once a new and different method of employing GPS patch antennas is considered. While most antenna designs tend to maximize the available FOV to a

given antenna for navigation and attitude determination purposes, a different approach is introduced in this paper which uses a reduced FOV.

Using multiple antennas distributed over the surface of a hemisphere, and restricting the FOV of each antenna to a predetermined cone can provide a workable solution. In this way, each antenna functions as a star tracker, whose "stars" are the GPS SVs themselves. Two such arrays of restricted FOV antennas still allow full sky coverage of the GPS constellation thereby permitting navigation solutions to be determined at any attitude. Since the nominal GPS navigation solution fixes the positions of the GPS SVs as well as the user vehicle in time and space, the sightlines from the user to the GPS constellation may also be determined. If each antenna can be "polled" to determine which GPS SVs are visible in each restricted FOV at a given time, information about where the known sightlines are relative to the antenna array is also possible. Finally, fixing the antenna geometry relative to the vehicle body 
frame allows vehicle attitude information to be determined from the orientation of multiple sightlines in the restricted FOVs of the antenna array.

\section{Sensor Configurations}

For the initial feasibility study, a six-element sensor array is employed. The computer model has each of the six antenna elements mounted to one face of a hemi-dodecahedron (see Figure 1). This configuration has the advantage of allowing one reference element to be oriented parallel to the sensor mounting plane, while maintaining a uniform separation between all adjacent antennas. For the initial study, the half-cone angle is 37.48 degrees, effectively dividing half of the sky into six overlapping FOVs, entirely covering half the sky while avoiding regions simultaneously observable by three elements. Again, for the sake of simplicity in this initial study, the sensor is assumed to be mounted to a LEO spacecraft which is directly over the north pole of the Earth, and oriented with a zero degree offset in both azimuth and elevation with respect to tile inertial frame (zero degree attitude error). The sensor has been presented with a representative scattering of GPS SVs in inertial space. A planar projection of the sensor FOV is shown in Figure 2, where the element centers are labeled as S0 through S5 and the GPS SVs are labeled as N01 through N31. In this projection, the heavy line represents the Earth limb, below which GPS SVs are eclipsed and may not be employed in subsequent calculations.

Several simple computer models were run, with successful attitude determination. Considerable improvements can be made by dividing the sky into a greater number of smaller areas. Maintaining the full sky coverage permitted by the hemi-dodecahedron design requires the inclusion of additional antenna elements. Spherical symmetry makes the buckeyball a very attractive geometry. A buckeyball is a solid which may be viewed as a combination of two regular solids, the dodecahedron and the icosohedron; upon the realization that a dodecahedron has 12 faces and 20 vertices, while the icosohedron has 20 faces and 12 vertices. A three dimensional fusion of the two solids renders a solid with 32 faces, 12 identical pentagons (half-cone angle of 20.07 degrees) regularly arranged as on a dodecahedron and 20 identical hexagons (half-cone angle of 23.8 degrees) arranged as are the triangles of an icosohedron. For the next series of feasibility studies, a 16-faced hemi-buckeyball sensor has been employed (see Figure 3), again with all the half-cone angles set to avoid regions simultaneously observable by three elements. The vehicle model was again assumed to be a LEO spacecraft at the north pole with the sensor aligned with the inertial reference frame (zero degree attitude error), as shown in Figure 4. 
Another way to divide the sky into a greater number of smaller areas without adding more sensor elements is to enlarge reduced field-of-views (RFOVs) in the basic design to create areas of overlap, using the information provided by the resulting overlapping coverage (see Figure 5, which has a $27 \%$ increase in the half-cone angles). The overlap regiops and remaining regions now yield effective FOVs (EFOV) for the sky coverage (the RFOV and EFOC are the same when no overlap occurs). Two hemibuckeyballs oriented in opposite directions considered as a single sensor can provide full $4 \pi$ steradian coverage of the sky. This orientation involves two hemi-buckeyballs mounted to the user spacecraft (e.g., one to the zenith deck and the other to the nadir deck). Not only does this configuration provide the cap . jility of two individual buckeyballs, but allows for the additional division of the full sky into areas where the two halves overlap. Regions covered by only one element correspond to the faces of the buckeyball (32), regions covered by two correspond to the edges (90) and, not surprisingly, regions covered by three elements correspond to the vertices of the buckeyball (60). For the full buckeyball, this divides the full sky up into 182 uniquely defined areas. A planar projection of this configuration is shown in Figure 6. The configurations considered in this study are summarized in Table 1 (EFOV-1 corresponds to regions covered by one element, and likewise for EFOV-2 and EFOV-3).

Table 1 Geometry and Configurations

\begin{tabular}{clccccc} 
CEGANS & \multicolumn{1}{c}{ Geometry } & Cov & EFOV-1 & EFOV-2 & EFOV-3 & Total Areas \\
\hline A1 & Full-Buckeyball & $4 \pi$ & 32 & 90 & 60 & 182 \\
A2 & Full-Buckeyball & $4 \pi$ & 32 & 90 & 0 & 12 ? \\
B1 & Hemi-Buckeyball & $2 \pi$ & 16 & 33 & 20 & 6 \\
B2 & Hemi-Buckeyball & $2 \pi$ & 16 & 33 & 0 & 49 \\
C1 & Full-Dodecahedron & $4 \pi$ & 12 & 30 & 20 & 62 \\
C2 & Full-Dodecahedron & $4 \pi$ & 12 & 30 & 0 & 42 \\
D1 & Hemi-Dodecahedron & $2 \pi$ & 6 & 10 & 5 & 21 \\
D2 & Hemi-Dodecahedron & $2 \pi$ & 6 & 10 & 0 & 16
\end{tabular}

\section{Case Studies}

To allow easy understanding of the environment during the sensor development phase, the first set of refinement studies have been executed assuming the CEGANS to be affixed to a static user spacecraft with a zero degree attitude error under a static GPS constellation. This allowed for realistic yet comprehensible results to be obtained, while providing a consistent comparative basis of results. Development of increasingly complex CEGANS types followed. Once the desired level of sensor 
complexity has been successfully modeled, refinements in the environmental model could be addressed. Up to this point, all attitude solutions are obtained assuming the CEGANS to be affixed to a static LEO (700 km altitude) user spacecraft with a zero degree attitude error under a static GPS constellation. Moving to the next level of complexity involves setting the user spacecraft in motion about the Earth. For this step, the user spacecraft is assumed to be an Earth pointing vehicle, with no attitude errors, maintaining the CEGANS in a zenith pointing orientation as the spacecraft moved under a static constellation. A polar orbit is used to provide the widest variety of geometries with respect to the GPS constellation.

In cich case, sightlines from the user spacecraft to each SV in the GPS constellation are determined, with those behind the Earth (from the user spacecraft's perspective) eliminated from subsequent consideration. The GPS SV sightlines are then compared to the boresight vectors (and cone angles) of each sensor element to establish which GPS SVs fall within the RFOV of each sensor element. In this way, a truth model can be developed while collecting the sightline data to be made available for attitude determination. This sightline data takes the form of a binary "visibility" matrix (1: SV visible, 0 : SV not visible) with GPS SVs along one axis and CEGANS element along the other. Since the nominal GPS navigation function permits inertial position determination of all the GPS SVs as well as the user spacecraft, vector differences allow determination of the vector sightlines from the user spacecraft body to the GPS SVs in the inertial frame. Comparing the GPS SV sightlines to the known geometry of the various CEGANS elements in the user spacecraft body frame through the visibility matrix allows determination of a unique attitude which permits the correct GPS SVs to be "seen" by the correct CEGANS elements at a particular time.

\section{Attitude Determination}

Once the body boresight vectors and spacecraft sightline vectors are given, then the attitude can be determined. This is accomplished by minimizing the following loss function (first posed by Wahba [15])

$$
J(A)=\frac{1}{2} \sum_{i=1}^{n} w_{i}\left\|\underline{b}_{i}-A \underline{s}_{i}\right\|^{2}
$$


where $\underline{b}_{i}$ now denotes $i^{\text {th }}$ unit vector to the center of the EFOV, $\underline{s}_{i}$ denotes the normalized $i^{\text {th }}$ sightline vector, and $w_{i}$ is a weighting factor. The optimal choice of weights will be discussed below. The error introduced using the new sensor configuration is mostly due to the incorrect knowledge of the actual line-of-sight to the GPS spacecraft in the body frame, since all visible GPS spacecraft in an antenna FOV are assumed to have a body vector in the center of the EFOV. It is possible to have overlapping circles so that that all EFOVs have approximately the same area. If the areas are equal for each corresponding boresight, then Equation (1) can be simplified by setting $w_{i}=1$. Once the weights have been chosen, then the solution for the attitude can be found using standard techniques that minimize Wahba's problem.

A simple solution for the attitude matrix in Equation (1) is given by performing a singular-valuedecomposition of the following matrix [17]

$$
F=\sum_{i=1}^{n} w_{i} \underline{b}_{i} \underline{s}_{i}^{T}=U \Sigma V^{T}
$$

The optimal solution for the attitude matrix is given by [17]

$$
A_{\text {opt }}=U_{+} V_{+}^{T}
$$

where

$$
\begin{aligned}
& U_{+}=U[\operatorname{diag}(1,1, \operatorname{det} U)] \\
& V_{+}=V[\operatorname{diag}(1,1, \operatorname{det} V)]
\end{aligned}
$$

The covariance of the estimation error angle vector in the body frame is given by

$$
E\left\{\underline{\delta \alpha} \underline{\delta \alpha}^{T}\right\} \equiv P=\left[\sum_{i=1}^{n}\left(\sigma_{b i}^{2}+\sigma_{s i}^{2}\right)^{-1}\right]^{-1}\left(I-F A_{o p t}^{T}\right)^{-1}
$$

where $\underline{\delta \alpha}$ corresponds to a small error angle, and $\sigma_{b i}$ and $\sigma_{s i}$ are the standard deviations of the body and sightline measurement error processes, respectively. Since the GPS spacecraft positions are well known, it is reasonable to assume that $\sigma_{b i} \gg \sigma_{s i}$ (for the remainder of the paper $\sigma_{i}^{2} \equiv \sigma_{b i}^{2}$ ). 
Since the $z$-axis of the sensor coordinate system is outward along the boresight, then the reconstructed unit vector in the body frame is given as a function of the co-elevation $\theta_{i}$ and azimuth $\phi_{i}$

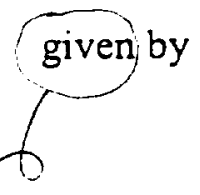

$$
\underline{b}_{i}=\left[\begin{array}{c}
\sin \theta_{i} \cos \phi_{i} \\
\sin \theta_{i} \sin \phi_{i} \\
\cos \theta_{i}
\end{array}\right]
$$

The true (error-free) unit vector is given by

$$
\underline{b}_{i}^{\text {rrue }}=\left[\begin{array}{l}
0 \\
0 \\
1
\end{array}\right]
$$

If the error distribution is axially symmetric about $\underline{b}_{i}^{\text {true }}$ (which is a reasonable assumption for the GPS sensor), then the variance of the body measurement process for a uniform distribution over a circle of radius $\rho_{i}$ can be determined by

$$
\sigma_{i}^{2}=\frac{1}{2} E\left\{\sin ^{2} \theta\right\}=\frac{\frac{1}{2} \int_{\rho_{i}}^{1}\left(1-\cos ^{2} \theta\right) d(\cos \theta)}{1-\cos \rho_{i}}
$$

which leads to

$$
\sigma_{i}^{2}=\frac{1}{6}\left(2+\cos \rho_{i}\right)\left(1-\cos \rho_{i}\right)
$$

Note that if $\rho_{i}$ is small, then the standard deviation can be accurately approximated by $\sigma_{i} \approx \rho_{i} / 2$.

Determining the optimal weights in Equation (1) is not straightforward. An intuitive approach uses $w_{i}=1 / \sigma_{i}^{2}$. Ignoring overlap regions, the error for each antenna encompasses a small circle on a curved surface of the unit sphere. The area of a small circle of angular radius $\rho$ is given by [16]

$$
\Pi=2 \pi(1-\cos \rho)
$$

Now consider the case where the FOV of two antennas overlap. The overlap area between two small circles of angular radii $\rho$ and $\zeta$, separated by a center-to-center distance $\beta$ is given by [16] 


$$
\begin{aligned}
\Omega=2 \pi & -2 \cos \rho \operatorname{acos}\left[\frac{\cos \varsigma-\cos \rho \cos \beta}{\sin \rho \sin \beta}\right]-2 \cos \varsigma \operatorname{acos}\left[\frac{\cos \rho-\cos \varsigma \cos \beta}{\sin \varsigma \sin \beta}\right] \\
& -2 \operatorname{acos}\left[\frac{\cos \beta-\cos \varsigma \cos \rho}{\sin \varsigma \sin \rho}\right] \quad(|\rho-\varsigma| \leq \beta \leq \rho+\varsigma)
\end{aligned}
$$

The overlap region can also be used to define another boresight vector. Suppose that two areas overlap, and each area has each center boresight vector given by $\underline{b}_{1}$ and $\underline{b}_{2}$. Then, the boresight vector of the overlap region is simply given by

$$
\underline{b}_{3}=\frac{\underline{b}_{1}+\underline{b}_{2}}{\left\|\underline{b}_{1}+\underline{b}_{2}\right\|}
$$

This allows another measurement set to be made available simply by overlapping the FOV of two antennas. Also, the non-overlapping part of antenna FOV area decreases simply by $\Pi-\Omega$. Choosing weights for the overlapping case becomes extremely difficult, since the error distribution is no longer uniform in general. Since this paper focuses on the application of the sensor and not on a purely theoretical analysis, a number of simplifications have been made. First, for the non-overlapping case, Equation (9) can be approximated by a solid angle given as the projected surface area divided by the total surface area of the sphere, so that

$$
\frac{1}{w_{i}}=\sigma_{i}^{2} \approx \frac{2 \pi(1-\cos \rho)}{4 \pi}=\frac{1}{2}(1-\cos \rho)
$$

This is a good approximation even for large values of $\rho$ (see Figure 7). Next, it is assumed that the same approximation holds true for the overlapping case; so that the weight for the overlapping region is given by $w_{i}=4 \pi / \Omega_{i}$, and the weight for the non-overlapping region is given by $w_{i}=4 \pi /\left(\Pi_{i}-\Omega_{i}\right)$. Therefore, as the area of the small circle decreases, more weight is placed on that measurement in the attitude determination, which intuitively makes sense. The case for triple overlaps becomes increasingly complex; however, for this study this case yields areas that are approximately equal so that Equation (13) is a good approximation for the EFOVs.

The performance of the attitude determination algorithm may be enhanced. This is accomplished by assuring that vectors formed by mapping the sightline vectors into the body frame (using the determined attitude) are within the corresponding antenna FOV centered at the assumed body-frame boresight vector. The procedure is as follows: 
1) Determine any overlap regions and corresponding boresight vectors.

2) Determine the optimal weights using area formulas.

3) Determine the available GPS spacecraft in each area and form sightline vectors.

4) Determine the attitude $(A)$ by minimizing Equation (1).

5) Map the sightline vectors into the body frame, i.e., $\underline{\hat{b}}_{i}=A \underline{s}_{i}$.

6) Determine the angle between the mapped vector and actual boresight $\vartheta_{i}=\operatorname{acos}\left(\underline{\underline{b}}_{i} \cdot \underline{b}_{i}\right)$.

7) Determine if each mapped vector $\underline{\hat{b}}_{i}$ is outside of its corresponding FOV.

If a mapped vector is not within its corresponding FOV, then the weight associated with the corresponding boresight vector and sightline vector should be decreased by some factor (e.g., $1 / 2$ ). The procedure is continued until all mapped vectors are within their corresponding FOVs. This ensures that the physical nature of the determined attitude is correct.

\section{Simulation Results}

In this section, simulation results are presented for a number of sensor configurations. The first test case involves a simulated non-moving spacecraft at the zenith position using the hemi-dodecahedron sensor (D2 in Table 1), as seen in Figure 2. There are nine available GPS sightlines with one overlapping SV in the S2 and S3 FOVs. With the weighting scheme developed in the previous section, it was determined that the found attitude provided mapped sightline vectors within their respected FOVs. Therefore, the attitude is a consistent with the sensor configuration. Attitude accuracy and $3 \sigma$ bounds using Equation (5) are shown in Table 2. Clearly the simple sensor approach provides a feasible method for attitude determination. The $3 \sigma$ bounds are large due to the assumption of a uniform error distribution, which results in an absolute worst case scenario (i.e., when all actual body measurements are at the sensor edge of view).

Table 2 Results for Case 1 (see Figure 2)

\begin{tabular}{|c|c|c|c|c|c|}
\hline \multicolumn{3}{|c|}{ Attitude Errors (deg) } & \multicolumn{3}{c|}{$3 \sigma$ bounds (deg) } \\
\hline Roll & Pitch & Yaw & Roll & Pitch & Yaw \\
\hline 1.47 & -3.42 & -6.48 & 22.8 & 24.7 & 20.8 \\
\hline
\end{tabular}


The second test case involves the same spacecraft at the zenith position using the hemi-buckeyball' $(B X £$ in Table 1), as seen in Figure 4. For this case, there are a total number 11 available GPS sightlines, with three overlapping spacecraft. In order to quantify the concept of using overlapping FOVs, two different solutions were determined. The first assumes that no overlapping occurs. Results for the attitude accuracy and $3 \sigma$ bounds are shown in Table 3. Clearly, decreasing the sensor FOV increases attitude accuracy (as expected). The next solution uses the overlapping regions, with an effective boresight centered in each overlapping region. Results for the attitude accuracy and $3 \sigma$ bounds are also shown in Table 3. Clearly attitude knowledge improves for yaw, but more importantly the $3 \sigma$ bounds are dramatically reduced. This shows that significant improvements are possible by considering the overlapping regions, with areas much less than the non-overlapping regions.

Table 3 Results for Case 2 (see Figure 4)

\begin{tabular}{|c|c|c|c|c|c|c|}
\cline { 2 - 7 } \multicolumn{1}{c|}{} & \multicolumn{3}{c|}{ Attitude Errors (deg) } & \multicolumn{3}{c|}{$3 \sigma$ bounds (deg) } \\
\cline { 2 - 7 } \multicolumn{1}{c|}{} & Roll & Pitch & Yaw & Roll & Pitch & Yaw \\
\hline non-overlap & -1.69 & 2.98 & -1.40 & 12.9 & 13.1 & 11.8 \\
\hline overtap & -1.60 & 2.32 & 0.81 & 4.91 & 6.3 & 4.8 \\
\hline
\end{tabular}

A dynamic test run has also been performed for a simulated Earth pointing spacecraft at one revolution per orbit (RPO). The sensor configuration is given by the full-buckeyball ( $A 1$ in Table 1). with increased half-cone angles, as seen in Figure 6. Increasing the half-cone angles results in approximately equal areas for the overlapping regions. The sensor measurements are sampled at 0.1 degree increments. A plot of the number of available GPS sightlines is shown in Figure 8. In general, the more available SV's the more accurate the attitude (the separation angle affects attitude accuracy as well). A plot of the attitude errors with $3 \sigma$ bounds is shown in Figure 9. Clearly, the theoretical weighting choice in Equation (20) provides accurate attitude error bounds. Also, the attitude errors are greatest when there are the fewest available number of SV's. For this sensor configuration case attitude accuracy within 5 degrees is possible. In order to further improve the accuracy a simple attitude filter has been implemented. This is a simple first-order Kalman filter that combines a propagated model with the determined attitudes. Since gyros are not used for this case, the angular velocity is assumed to be perfect (i.e., given by the one revolution-per-orbit motion). This assumption is not exact, since external disturbances and control errors are present in the actual system. These general involve dynamic coupling in the rollyaw axis for Earth pointing spacecraft, which are modeled by adding a bias to the pitch rate 
and sine wave to the roll and yaw axes with a 90 degree phase difference (see Ref. [18] for details). The simple filter is given by

$$
\begin{gathered}
\underline{\hat{q}}_{k+1}(-)=\exp \left\{\frac{1}{2} \Omega(\underline{\omega}) \Delta t\right\} \underline{\underline{q}}_{k}(+) \\
\underline{\hat{q}}_{k}(+)=(1-\alpha) \underline{\hat{q}}_{k}(-)+\alpha \underline{\tilde{q}}_{k}
\end{gathered}
$$

where $\Delta t$ is the sampling interval in seconds, $\underline{\tilde{q}}_{k}$ is the determined attitude at time $t_{k}, \underline{\hat{q}}_{k}$ is the estimated attitude at time $t_{k}$, and $\alpha$ is a scalar gain. This gain can be determined by minimizing the attitude errors from the simulated runs. A value that is too small adds too much model correction, and tends to neglect measurements. A value that is too large adds too much measurement noise, and tends to neglect model corrections. A value of $\alpha=0.1$ was determined to be optimal. Also, the steady-state attitude errorcovariance is given by [18]

$$
\hat{P}=\frac{\alpha}{2-\alpha} P
$$

A plot of the attitude errors and $3 \sigma$ bounds using the simple filter is shown in Figure 10 . Clearly, the attitude accuracy can be improved by nearly a factor of four. This simulation case clearly indicates that attitude determination using the simple sensor scheme is viable.

\section{Conclusions}

The concept behind the CEGANS sensor was presented and the theory of operation developed. Theoretical results were obtained and presented which are sufficient to demonstrate the feasibility of the CEGANS concept as viable means of providing an autonomous on-board attitude determination capability using GPS. The traditional interferometric method requires long baselines (on the order of a meter or more) to be effective, thereby limiting the size of the vehicle upon which it can be employed. Furthermore, the interferometric method can be difficult to initialize, and requires very accurate measurements of GPS SV carrier phase which can be exceedingly sensitive to multipath interference. These problems tend to impose geometric design and cumbersome testing requirements on the vehicle to provide clear, unobstructed, multipath-free sightlines to antennas distributed all over the vehicle, which depending on the vehicle may or may not be easily accommodated. Accuracies obtained herein prove that the CEGANS concept can meet the attitude requirements of a wide variety of vehicles. As technology evolves, GPS receivers and antennas become more and more capable, allowing for further 
refinement of this method by either better processing schemes or still greater number of antenna elements. This is in stark contrast to the potential for growth inherent in differential or carrier-phased base methods of comparable capability which are asymptotically approaching the limits imposed by physics.

\section{References}

[1] Elrod, B.D., and Van Dierendonck, A.J., "Pseudolites," Global Positioning System: Theory and Applications, Volume 2, edited by B.W. Parkinson and J.J. Spilker, Progress in Astronautics and Aeronautics, Vol. 164, AIAA, Washington, DC, 1996, Chapter 2.

[2] Parkinson, B.W., "Differential GPS," Global Positioning System: Theory and Applications, Volume 2, edited by B.W. Parkinson and J.J. Spilker, Progress in Astronautics and Aeronautics, Vol. 164, AIAA, Washington, DC, 1996, Chapter 1 .

[3] Cretaux. J.F., "Investigation of Orbit Determination using the GPS Constellation," Proceedings of the AAS/GSFC International Symposium on Space Flight Dynamics, Volume 1 (Greenbelt, MD), NASAGoddard, Greenbelt, MD, April 1993, AAS \#93-271.

[4] Haines et. al., "A Novel Use of GPS for Determining the Orbit of a Geosynchronous Satellite: The TDRS/GPS Tracking Demonstration" Proceedings of the 1994 ION-GPS (Salt Lake City, UT), The Institute of Navigation, Alexandria, VA, Sept. 1994, pp. 191-202.

[5] Axelrad, P., and Ward, L.M., "Spacecraft Attitude Estimation Using the Global Positioning System: Methodology and Results for RADCAL," AIAA Journal of Guidance, Control, and Dynamics, Vol. 19, No. 6, Nov.-Dec. 1996, pp. 1201-1209.

[6] Brock et. al., "GPS Attitude Determination and Navigation Flight Experiment," Proceedings of the 1995 ION-GPS (Palm Springs, CA), The Institute of Navigation, Alexandria, VA, Sept. 1995.

[7] Lightsey et. al. "Flight Results of GPS-Based Attitude Control on the REX-II Spacecraft," Proceedings of the 1996 ION-GPS (Kansas City, MO), The Institute of Navigation, Alexandria, VA, Sept. 1996, pp. 1037-1046.

[8] Cohen, C.E., “Attitude Determination Using GPS," Ph.D. Dissertation, Stanford University, Dec. 1992. 
[9] Cohen, C.E., "Attitude Determination," Global Positioning System: Theory and Applications, Volume 2, edited by B.W. Parkinson and J.J. Spilker, Progress in Astronautics and Aeronautics, Vol. 164, AIAA, Washington, DC, 1996, Chapter 19.

[10] Braasch, M.S., "Multipath Effects," Global Positioning System: Theory and Applications, Volume 1, edited by B.W. Parkinson and J.J. Spilker, Progress in Astronautics and Aeronautics, Vol. 164, AIAA, Washington, DC, 1996, Chapter 14.

[11] Cohen, C.E., and Parkinson, B.W., "Integer Ambiguity Resolution of the GPS Carrier for Spacecraft Attitude Determination," Advances in the Astronautical Sciences, Vol. 78, AAS \#92-015, pp. 107-118.

[12] Crassidis, J.L., and Markley, F.L., "New Algorithm for Attitude Determination Using Global Positioning System Signals," AIAA Journal of Guidance, Control, and Dynamics, Vol. 20, No. 5, Sept.Oct. 1997, pp. 891-896.

[13] Bar-Itzhack, I.Y., Montgomery, P.Y., and Garrick, J.C., "Algorithms for Attitude Determination Using GPS," Proceedings of the AIAA Guidance, Navigation, and Control Conference, New Orleans, LA), AIAA, Reston, VA, Aug. 1997, AIAA \#97-3616, pp. 841-851.

[14] Crassidis, J.L., Lightsey, E.G., and Markley, F.L., "Efficient and Optimal Attitude Determination Using Recursive Global Positioning System Signal Operations," submitted to the Journal of Guidance, Control and Dynamics.

[15] Wahba, G., "A. Least Squares Estimate of Spacecraft Attitude," Problem 65-1, SIAM Review, Vol. 7, No. 3, July 1965, p. 409.

[16] Wertz, J.R., "Spherical Geometry," Spacecraft Attitude Determination and Control, edited by J.R. Wertz, D. Reidel Publishing Co., Dordrecht, The Netherlands, 1978, pp. 727-736.

[17] Markley, F.L., "Attitude Determination Using Vector Observations and the Singular Value Decomposition," The Journal of the Astronautical Sciences, Vol. 36, No. 3, July-Sept. 1988, pp. 245258.

[18] Crassidis, J.L., Markley, F.L., Kyle, A.M., and Kull, K., "Attitude Determination Improvements for GOES," Proceedings of the Flight Mechanics/Estimation Theory Symposium (Greenbelt, MD), NASAGoddard, Greenbelt, MD, April 1993, pp. 151-165. 
Fig. 1 Spherically Symmetric (Hemi-Dodecahedron) Array of FOV Restricted Patch Antennas

Fig. 2 2-D Projection of the Hemi-Dodecahedron Sensor FOV

Fig. 3 A Hemi-Buckeyball

Fig. 4 2-D Projection of the Hemi-Buckeyball Sensor FOV

Fig. 5 2-D Projection of the Hemi-Buckeyball Sensor FOV with Increased Half-Cone Angles

Fig. 6 2-D Projection of the Full-Buckeyball Sensor FOV with Increased Half-Cone Angles

Fig. 7 Actual and Approximate Inverse Weighting

Fig. 8 Number of Available GPS Spacecraft

Fig. 9 Attitude Errors and 3 $\sigma$ bounds

Fig. 10 Attitude Errors and $3 \sigma$ bounds using a Simple Filter 


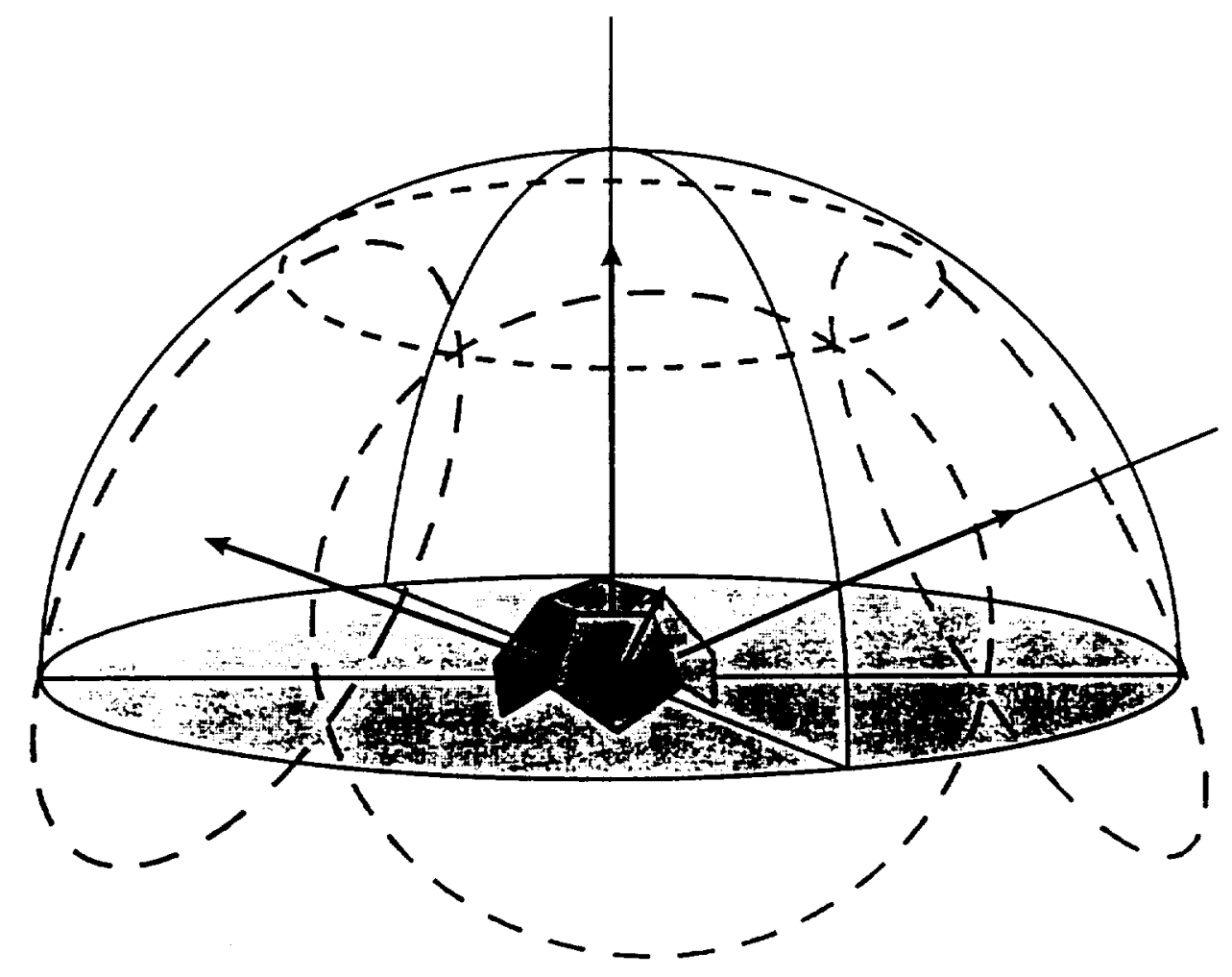




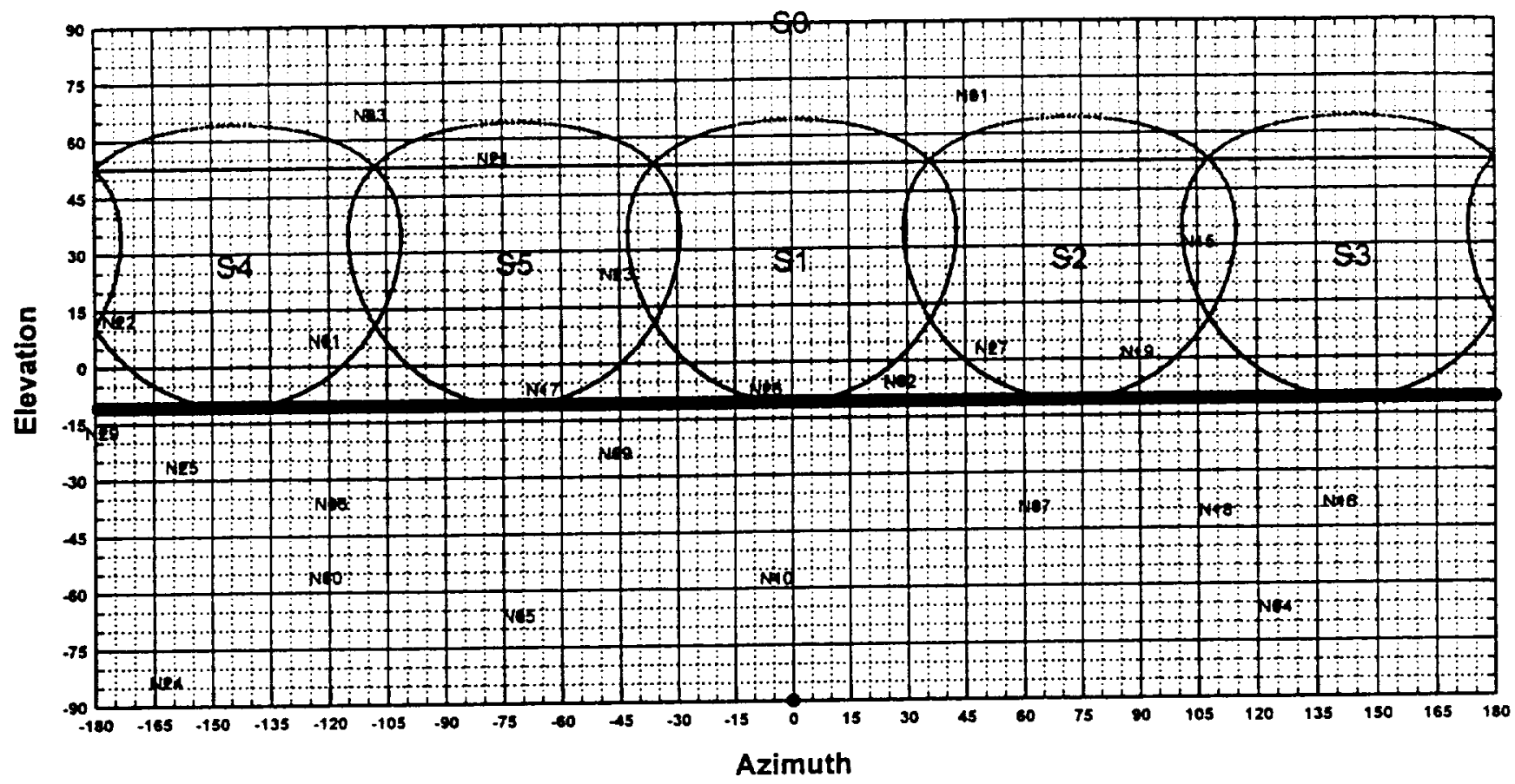




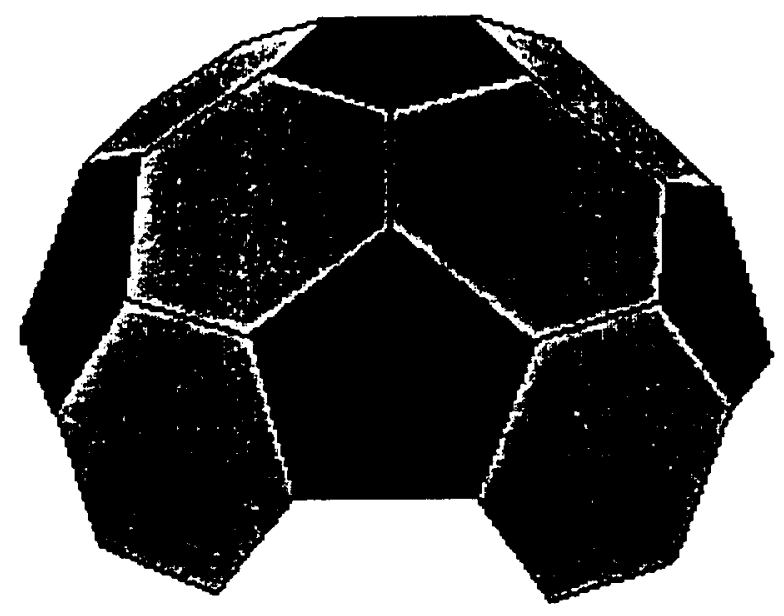

3 


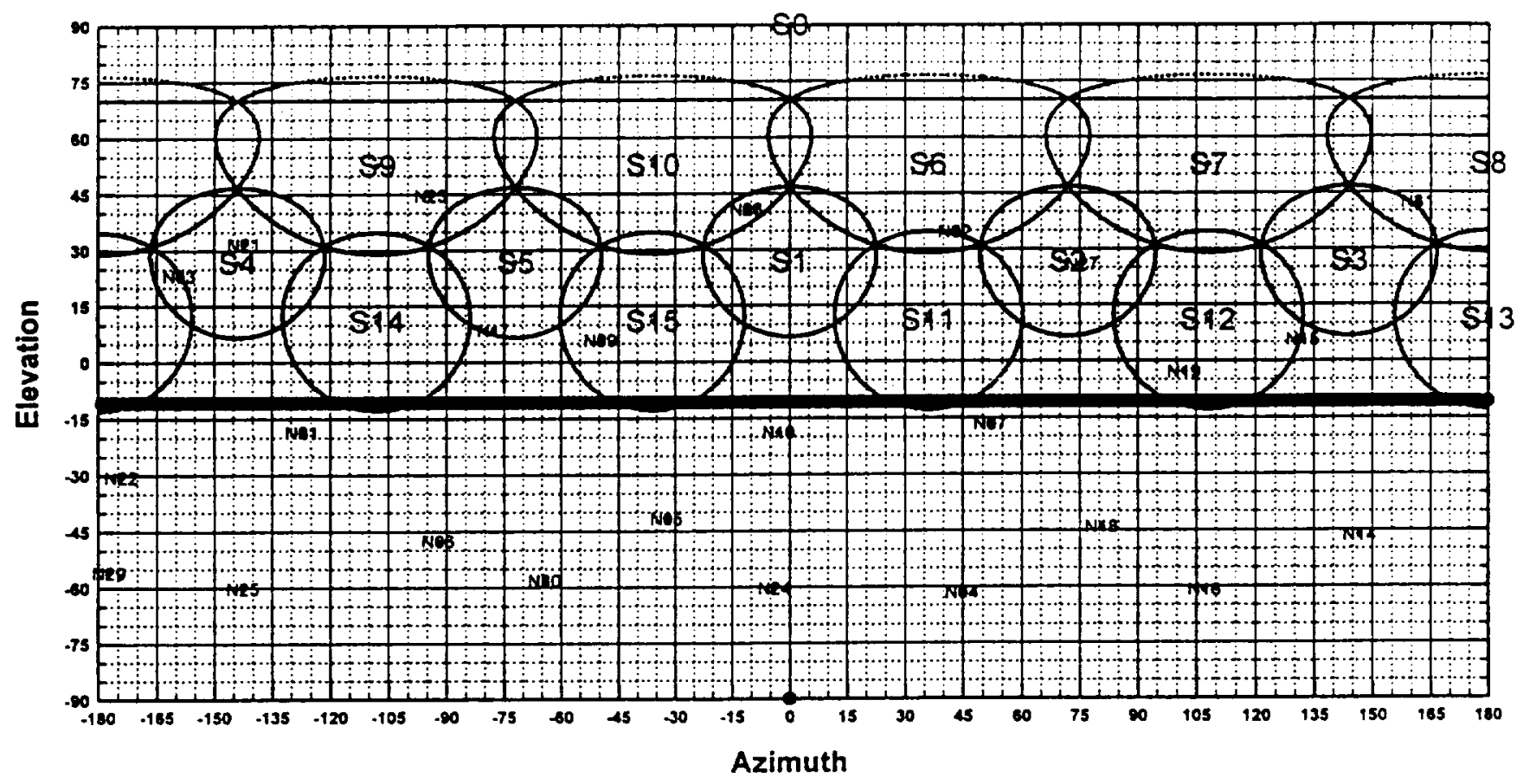




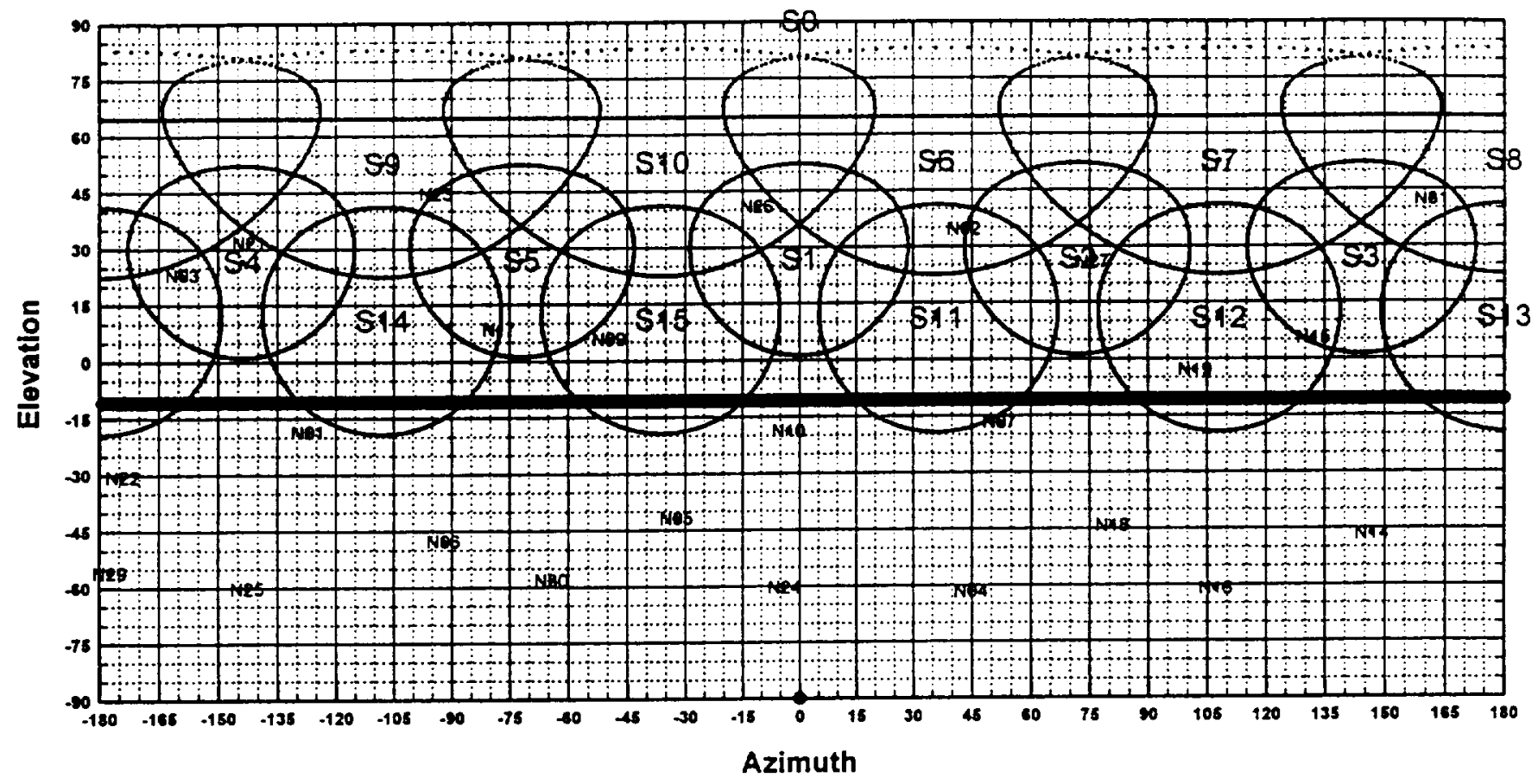




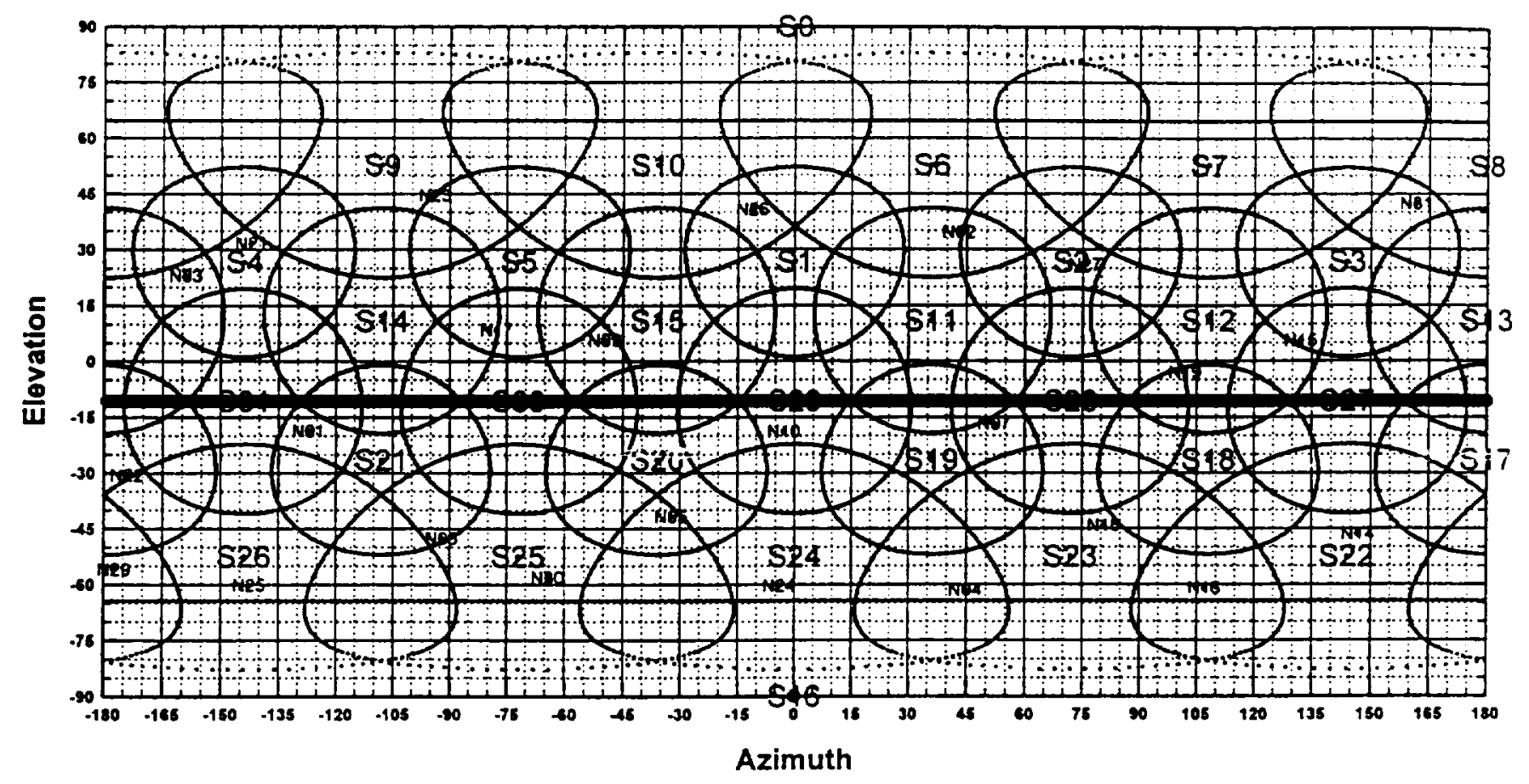




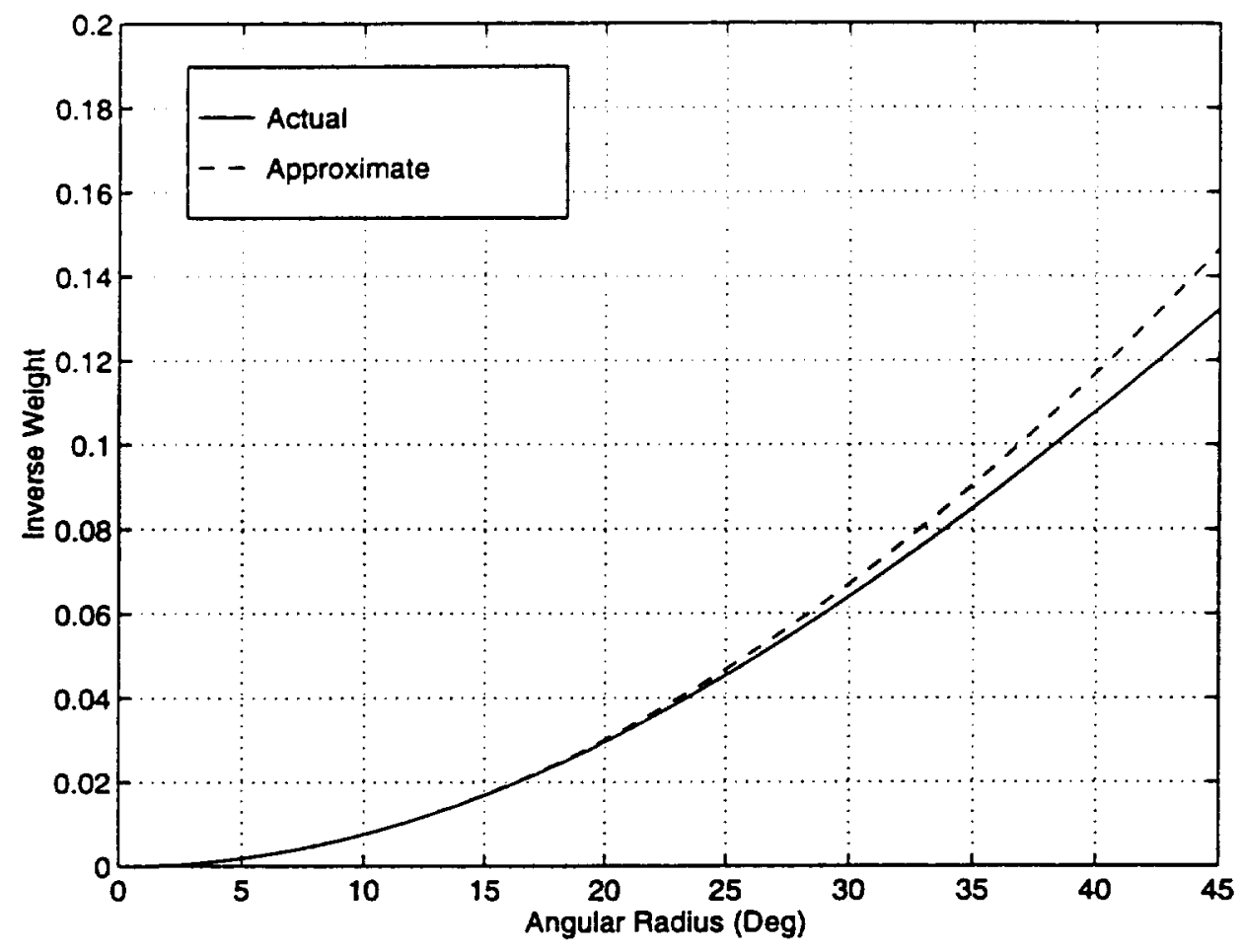




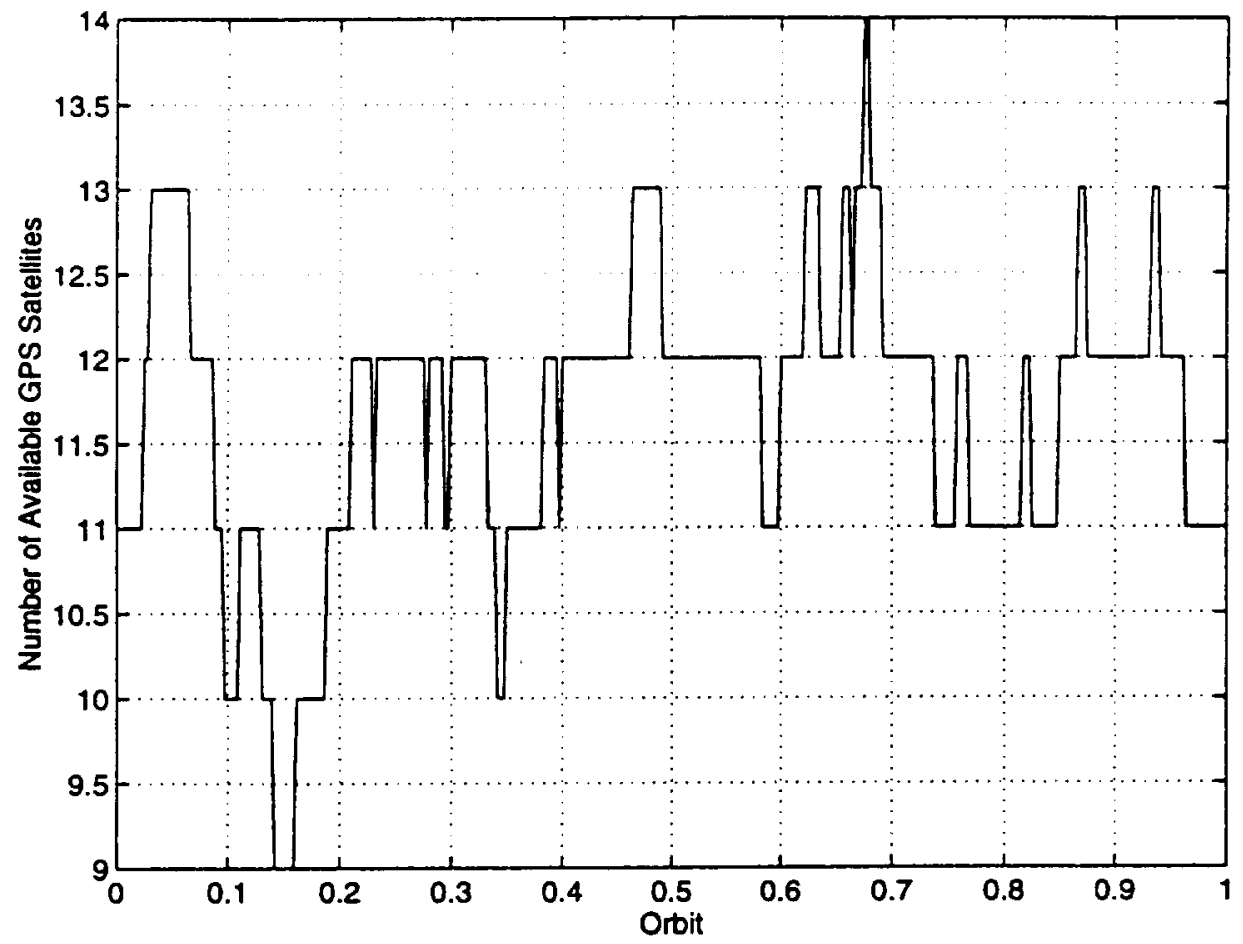



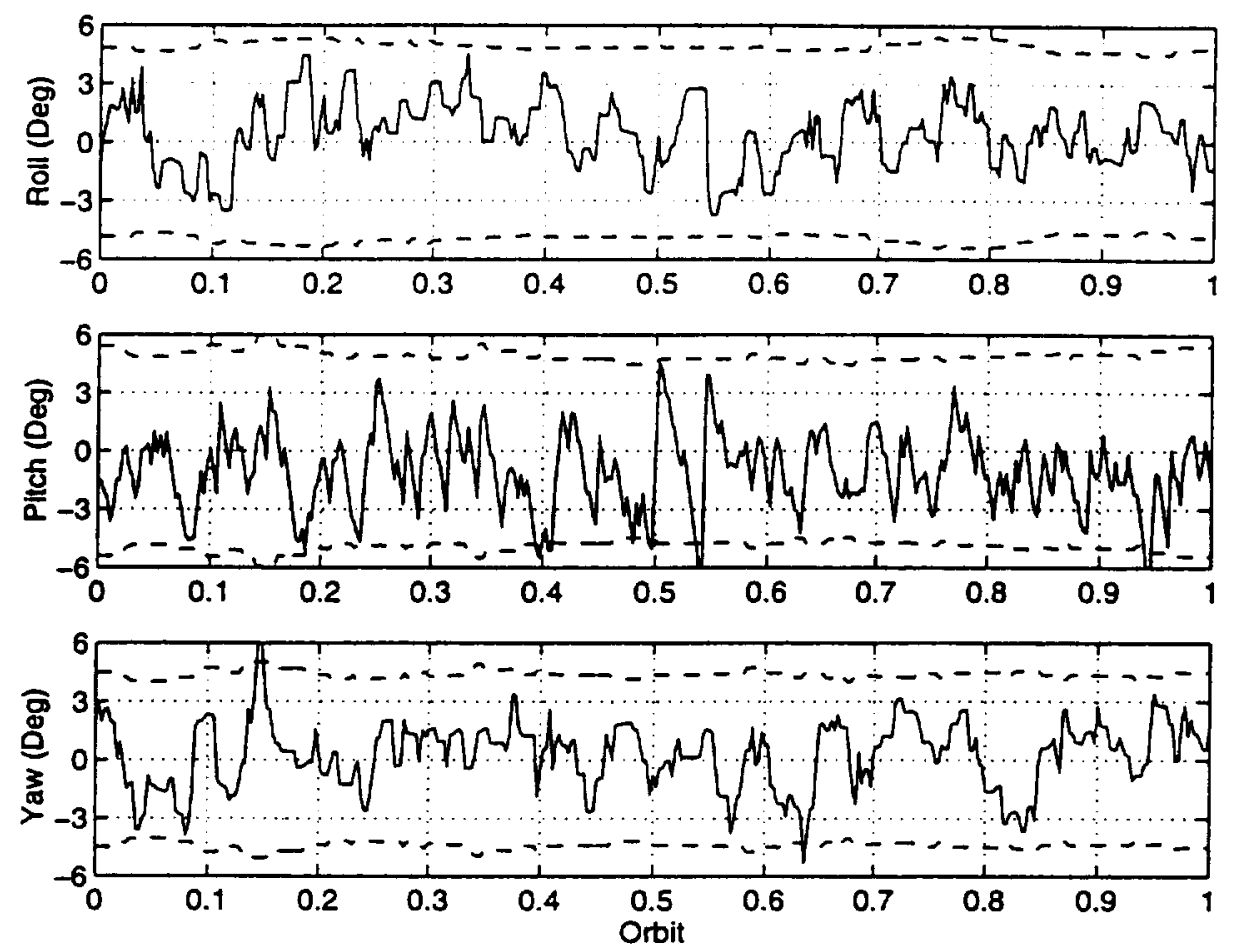

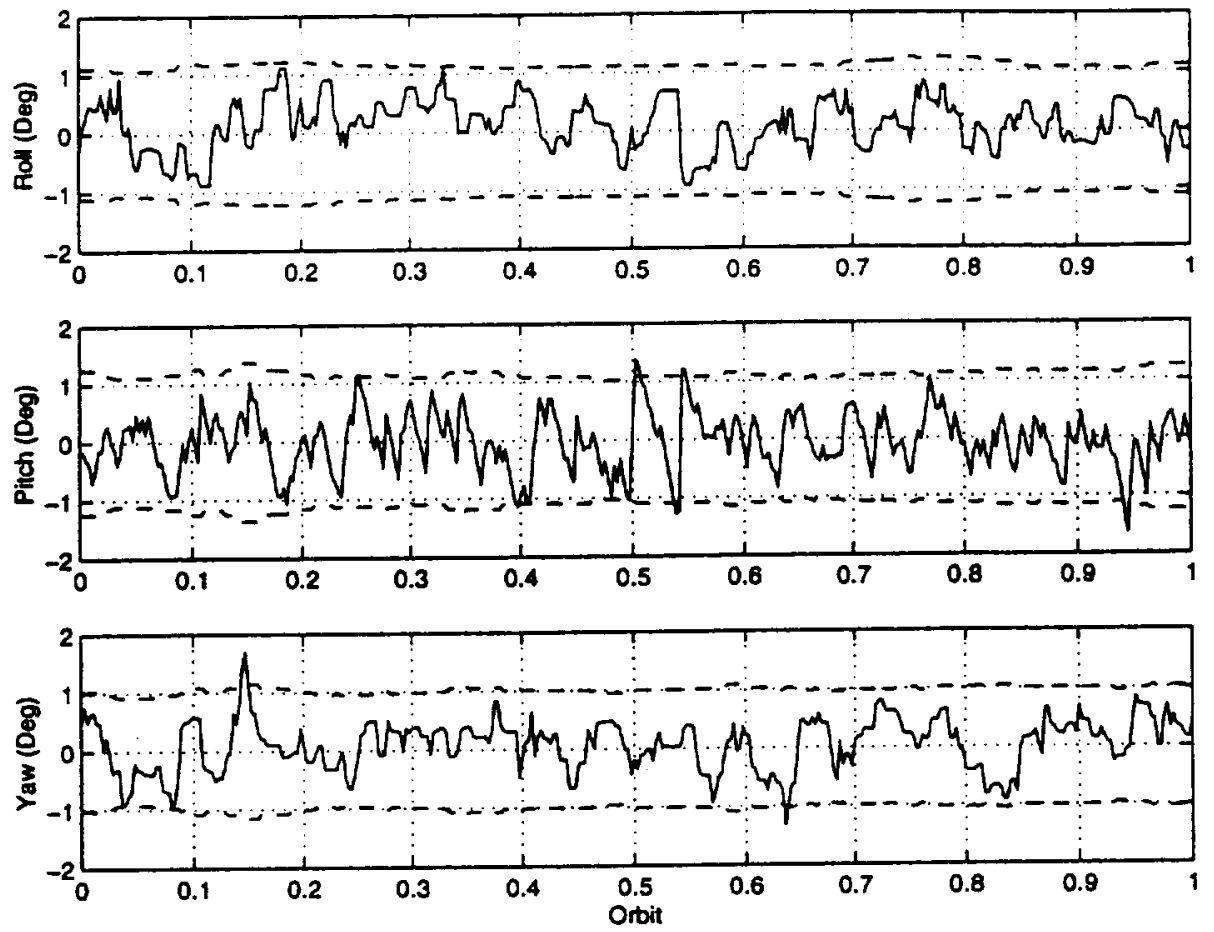